\title{
Experimental Study on Variation Strategies for Complex Social Pedestrian Groups in Conflict Conditions
}

\author{
Xiaolei Zou ${ }^{1}$, Xiaoyi $\mathbf{Q u}^{2}$, Ruihua $\mathrm{Xu}^{3}$ \\ ${ }^{1}$ College of Transportation Engineering, Tongji University \\ 4800\# Cao'an Road, Shanghai, China \\ zouxiaolei@tongji.edu.cn \\ ${ }^{2}$ College of Public Safety Management, Shanghai Open University \\ 288\# Guoshun Road, Shanghai, China \\ quxy@shtvu.edu.cn \\ ${ }^{3}$ College of Transportation Engineering, Tongji University \\ 4800\# Cao'an Road, Shanghai, China \\ zouxiaolei@tongji.edu.cn \\ rhxu@tongji.edu.cn
}

\begin{abstract}
The paper concentrates on an experimental study of the variation strategies of complex social pedestrian groups in conflict conditions. We tracked the trajectories of group members and analysed the configuration of both the complex group and its subgroups when the groups walked through a narrowing passage, passed by an obstacle or faced counter flows. We summarized the variation strategies of complex groups when they faced these conflict conditions. The effect of groups on the crowd was also studied. It was found that groups could have significant effect on self-organization of the crowd. The results in the paper could be applied in modelling pedestrian group decision and behaviour and analysing crowd dynamics.
\end{abstract}

Keywords: variation strategies, complex social pedestrian groups, group behavior, self-organization, conflict conditions

\section{Introduction}

In our previous studies, complex social pedestrian groups with more than 5 members are proved to be composed of simple subgroups with 2-3 members [1] and the complex groups always keep regular structures in free pedestrian flow [2]. The studies of other researchers also showed that facts $[3,4]$. But how do the groups variate and reorganize when they face conflict conditions? In this paper, we made a further experimental study on variation strategies for complex social pedestrian groups.

The paper concentrates on the variation strategies of complex social pedestrian groups in conflict conditions. We summarized the variation strategies of complex groups when they walked through a narrowing passage, passed by an obstacle or faced counter flows. We also studied the effect of groups on the crowd and found that groups could have significant effect on self-organization of the crowd.

\section{Controlled Experiments on Group Behavior}

From 2014 to 2016, we conducted a series of experiments on social pedestrian group behavior in Tongji University, Shanghai. Some of the experiments took place on October 27th and 28th with 60 student participants divided into complex social groups with 5-7 members in the following scenes:

(1) Group behavior and dynamics of crowd evacuation in the narrowing passage

In order to observe and test the behavior of complex groups with 5-7 members, the groups were asked to walk through a passage, the length of which was $8 \mathrm{~m}$ and the width gradually narrowed from $3 \mathrm{~m}$ to $1.5 \mathrm{~m}$. The groups kept walking for 5 meters after they passed the bottle neck. The scenes of both single groups and the crowd walking through the passage were conducted to observe the intragroup behaviour and intergroup interactions, which is shown in Fig.1. 


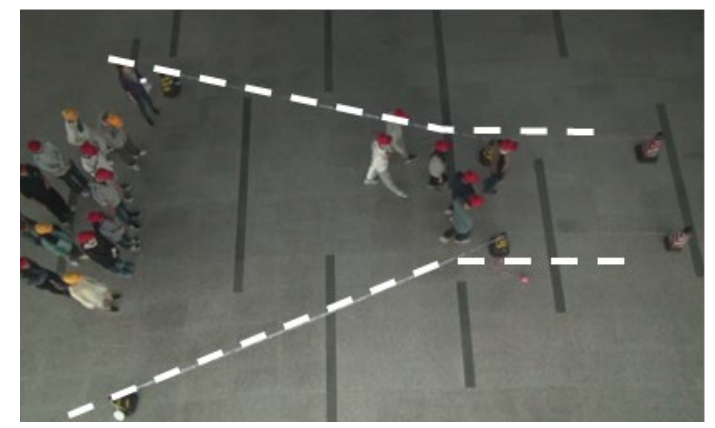

(a) Scene of a single group

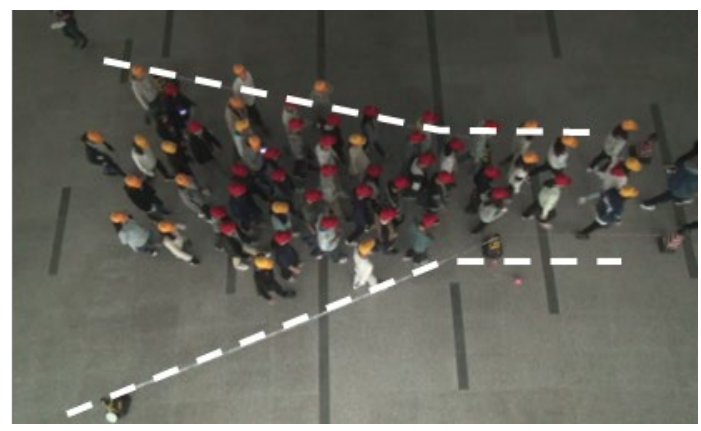

(b) Scene of the crowd

Fig. 1: Scenes of groups and crowds walking through the narrowing passage and bottle neck

(2) Obstacle avoidance behavior of groups and crowds

In this experiment, $3 \mathrm{~m}, 1.5 \mathrm{~m}$ and $0.8 \mathrm{~m}$ wide obstacles were set separately in a $6 \mathrm{~m}$ wide passage. Groups with 5-7 members were asked to walk through the passage in sequence to test the strategies of the obstacle avoidance behavior of the groups. In order to test the mutual influence of groups, crowds with groups were asked to go through the passage as well. See Fig. 2.

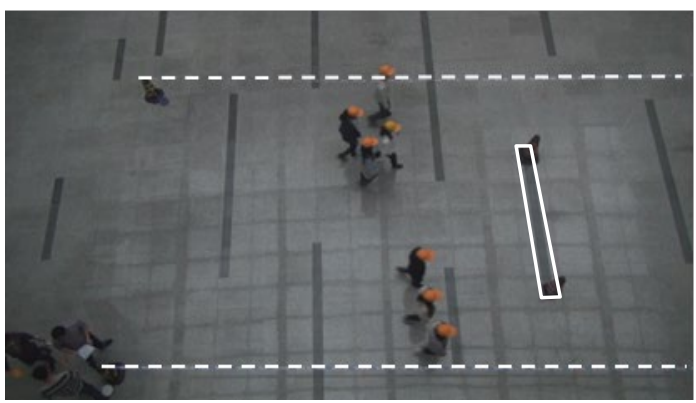

(a) A single group passing by the obstacle

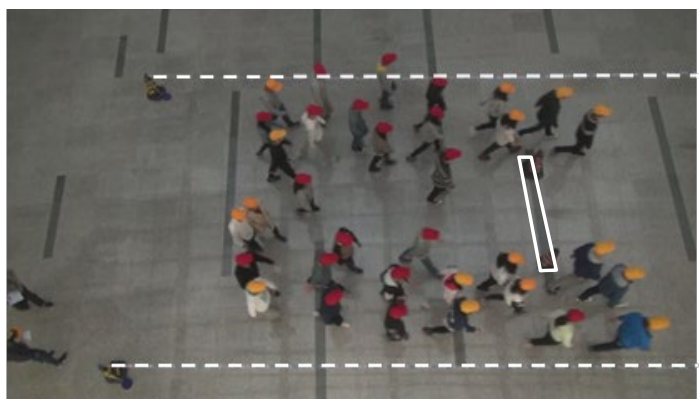

(b) The crowd passing by the obstacle

Fig. 2: Experiments for obstacle avoidance behavior of groups and crowds

(3) Group behavior in bidirectional counter flows

The width of the passage was set to $6 \mathrm{~m}$ and $3 \mathrm{~m}$, and groups with 5-7 members were asked to walk through the passage oppositely in sequence to test the variation strategies of the groups when they passed by each other. Bidirectional counter flows with groups and without groups were also asked to walk through the passage to test the impact of group behaviour on the evacuation of bidirectional counter flows. In addition, experiments for two vertical conflict streams walking across each other and bidirectional counter flows walking across the bottle neck were conducted as well.
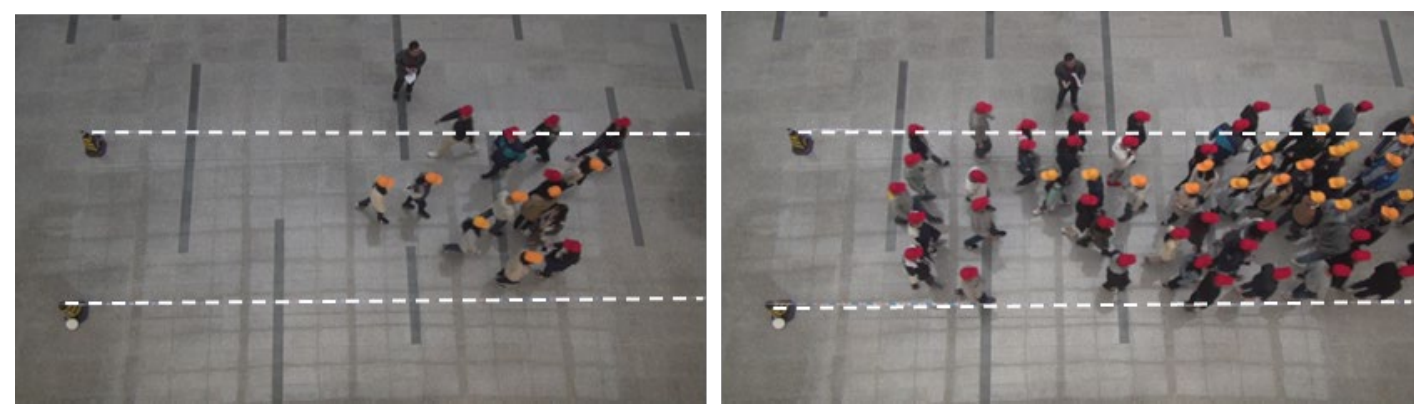
(a) Two groups passing by each other

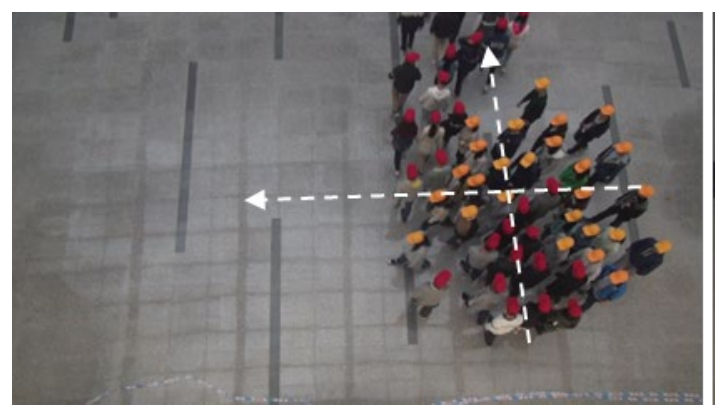

(b) Bidirectional counter flows in the passage

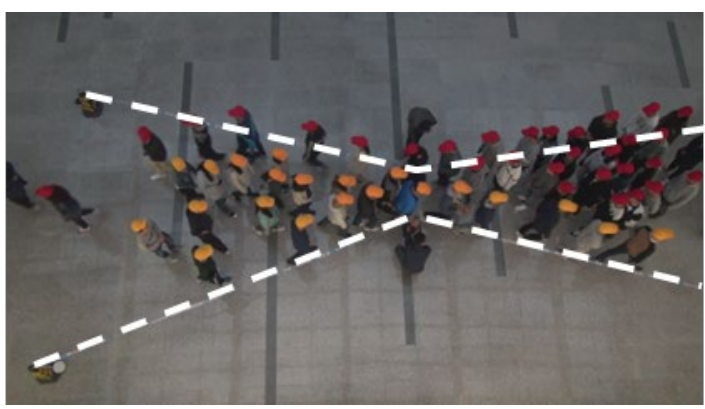

(c) Vertical conflict flows walking across each other (d) Bidirectional counter flows in the bottle neck Fig. 3: Experiments for group and crowd behavior in conflict conditions

\section{Strategies for Variation of groups in Narrow Space and Conflict}

We developed a visualization tool for group structure and trajectory analysis. By tracking and analysing the movement of the groups when passing narrowing passage and avoiding static obstacles, we summarized the variation strategies for group patterns.

\subsection{A visualization tool for group structure and trajectory analysis}

The visualization tool for group structure and trajectory analysis was developed in the environment of Visual Studio with C\# and Python. It provides a visualized way for observing the structure variation of moving complex groups and calibrating the group structure, and also the functions for speed and spatial distribution analysis for group members.

As shown in Fig. 4, trajectories of all the members of a complex group can be drawn. After setting the relationships of the members and subgroups in several key frames, the logical structure of the group for each moment can be expressed with colored dots and lines. The logical structure includes the location and connection of members, the barycenter of the complex group and subgroups, as well as the connection of subgroups. The connections between group members and subgroups are expressed with symbol expressions (See Reference [2]). All of the expressions and spatial locations of members can be deduced automatically and stored in time series.
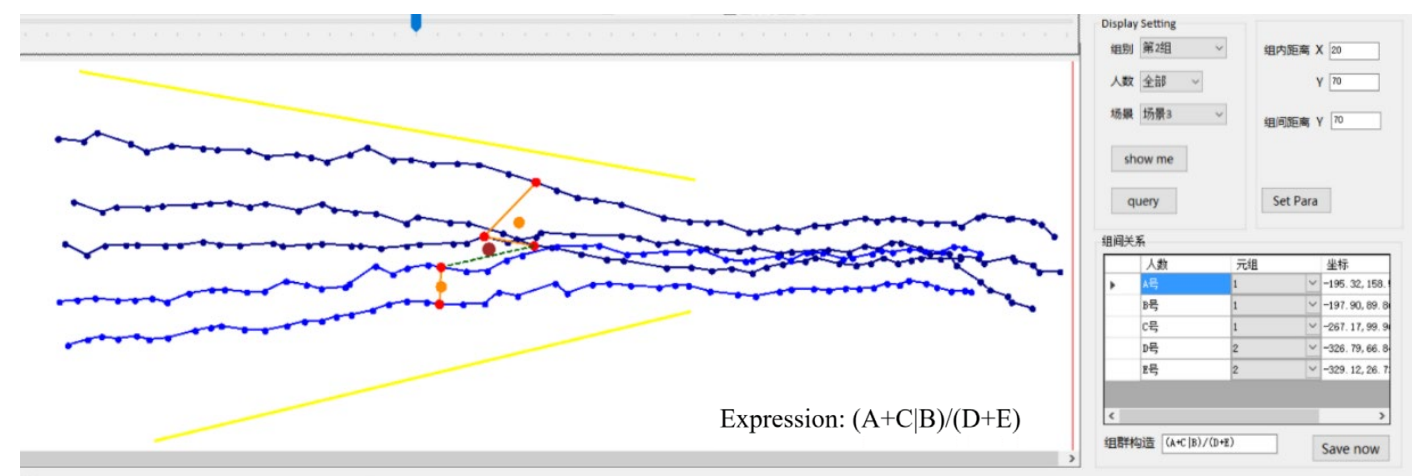

Fig. 4: A screen shot for the tool for dynamics visualizing analysis

\subsection{Strategies for Variation of Groups When Passing Narrowing Passage}

In scene 1, the motion of 61 complex groups with 5 members were tracked. The groups started walking in a free pattern (see Reference [2]) with their members aligned. We numbered the members from left to right in the group and plot the trajectories of all members of the tested groups together into one figure and plot the trajectories of all subgroups together into another. Fig. 5 shows that the groups experience the 
process of free walking, space compression, congestion and expansion during passing through the narrowing passage. In the state of free walking, boundaries of the distribution of group members are relatively clear, which means that group members intend to keep a comfortable private space from $50-60 \mathrm{~cm}$ in free walking. It is entirely consistent with our earlier research [5]. In other states, the distribution areas of the members overlap, which means that the structure and configuration of the groups vary in order to keep the private space of the members. Fig. 6 shows that the trajectories of subgroups align parallel to the walking direction when the passage is wide enough and overlap others when the passage becomes narrow.

Fig. 5 and Fig. 6 tell that both the group members and the subgroups vary their position in the group to adapt to the width of passage. What strategies for variation do groups adopt when the space becomes limited and how do they reorganized after they go out of the bottleneck?

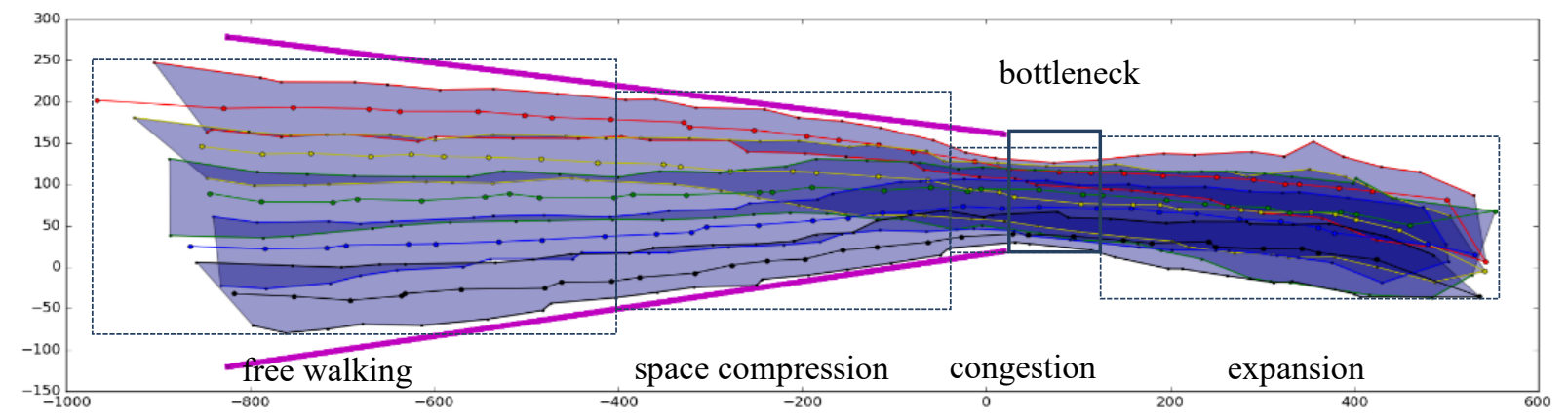

Fig. 5: Distribution of group members during passing through the narrowing passage

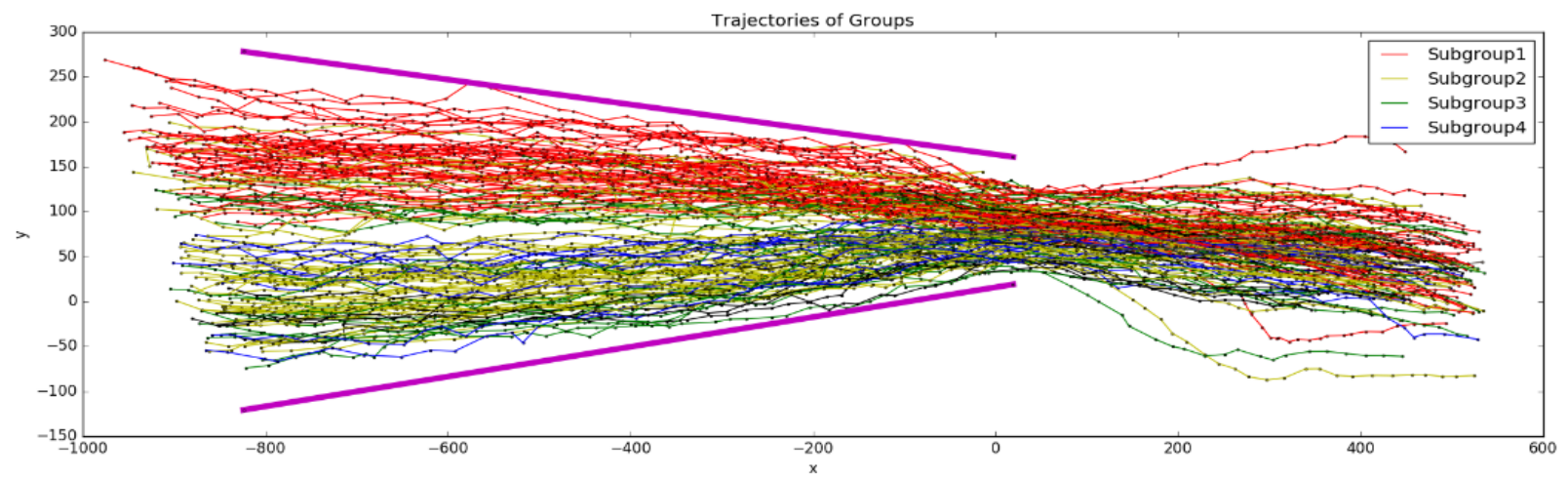

Fig. 6: Trajectories of subgroups

By analysing the trajectories of the members and social connections of them, we estimated the strategies of variation of the groups in two aspects, the constancy of the composition of members and the stability of the configuration of the groups.

At the beginning, the complex groups are naturally composed with subgroups and individuals. Approximately $63.9 \%$ of the complex groups are composed of 2 subgroups, which is the common structure of the 5-member groups (see Reference [1]). And 85.2\% of the complex groups spread in free pattern and simple pattern (see Reference [2]). The structures and patterns of the groups are consistent with the natural group features observed in our previous field studies from 2014 to 2016 in the massive long-distance striding (see Reference [2]).

While the groups walk from the wide side to the narrow side as shown in Fig. 1(a), the vertical space to the walking direction space narrows and most of the groups keep constant subgroup members, with a proportion of $77.1 \%$ of the tested ones. The constant groups adopt two main strategies. One is giving priority to keep the subgroup configuration by maintaining stable relative positions of members within the subgroup but changing the relative positions of the subgroups, and the other is giving priority to keep the complex group configuration by maintaining stable relative position of the subgroups within the complex 
group but changing the configuration of its subgroups. The data shows that $70.2 \%$ of the constant groups preferred to keep stable configurations of subgroups, which makes $54.1 \%$ of all the complex groups.

Fig. 7(a) shows one of the complex groups with 2 subgroups walking through the passage using strategy of keeping subgroup configuration. None of the subgroups changes its structure or configuration when it arrives at the bottle neck. The expressions for group members remains $(A+B)$ and $(C+D+E)$. But one subgroup accelerates while the other decelerates. The expression for subgroups changes from $a+b$ to $a \mid b$. The whole complex group keeps its configuration stable after it go out of the bottleneck.

Fig. 7(b) shows one of the complex groups with 2 subgroups walking through the passage using strategy of keeping the complex group configuration. The two subgroups walk at the same speed and keep the configuration stable when they arrive at and go out of the bottleneck with a symbol expression of $a+b$. But the subgroups compress their space and change the configuration.

After walking out of the bottleneck, the complex groups have enough space and can walk freely. However, most of them do not reorganize to their original structure but keep their new configuration. 84.8\% of the groups who use strategy of keeping subgroup configuration do not reorganize. They walk with a simple pattern with 2 rows and keep the whole group walking freely and the subgroup members communicating easily. And $71.4 \%$ of the groups who use strategy of keeping the complex group configuration do not reorganize but most of them spread to a free pattern to keep enough space for walking and communication.

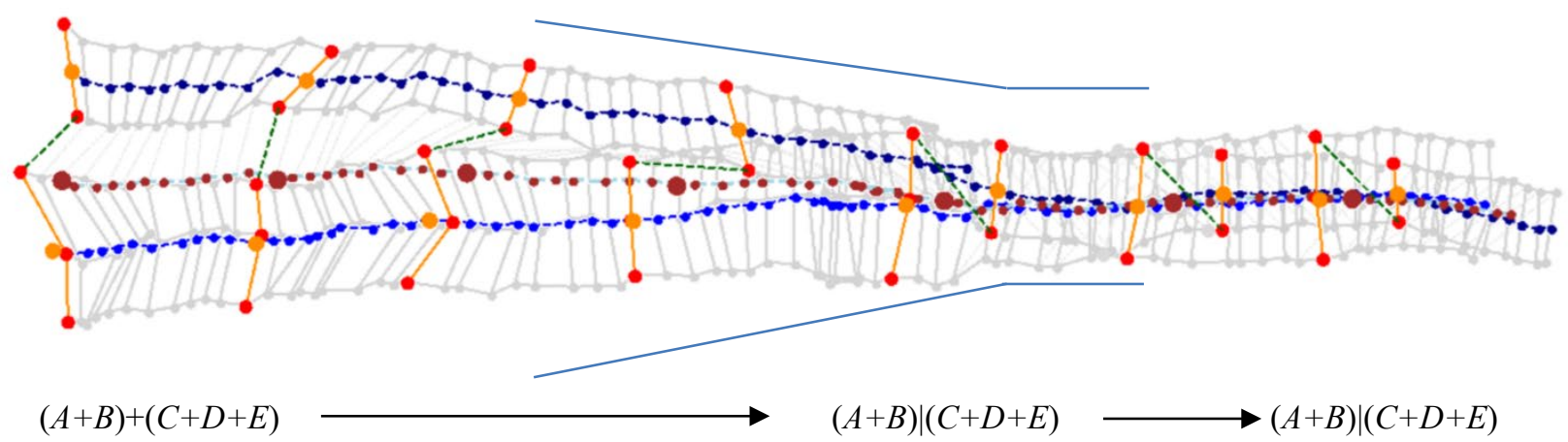

(a) Strategy of keeping subgroup configuration

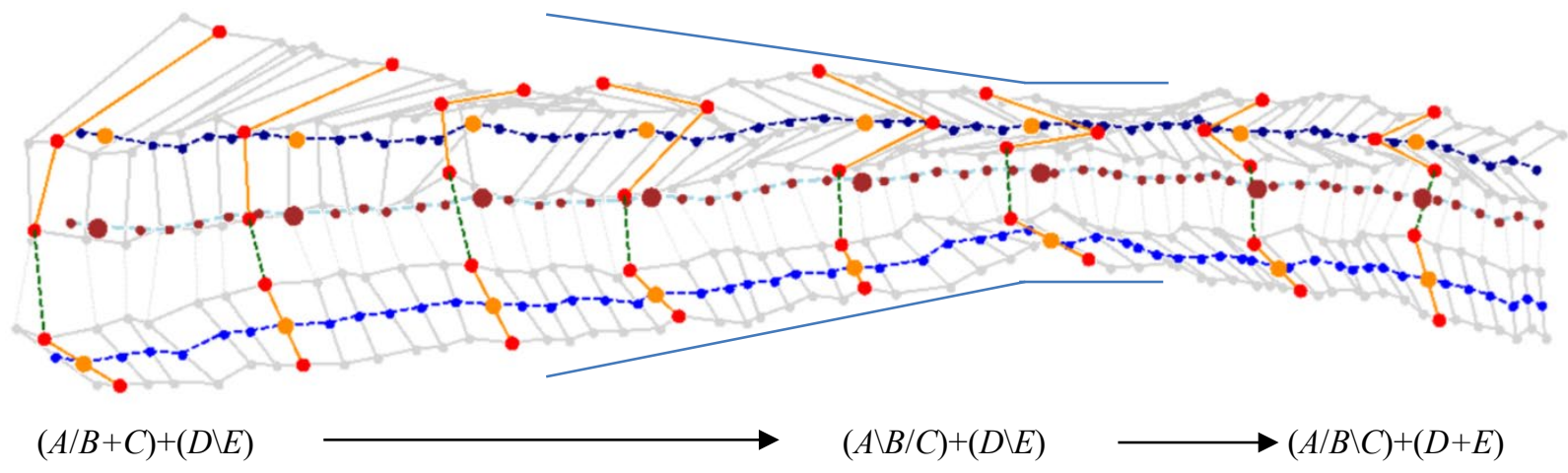

(b) Strategy of keeping complex group configuration

Fig.7: Variation strategies in narrow passages

\subsection{Strategies for Variation of Groups When Avoiding Obstacles}

Motion of 66 complex groups with 5 members were tracked by the tool in scene 2. In the experiments, two common initial patterns (See Reference [2]) of the groups were considered, the free pattern and the 
simple pattern. In free pattern, the group members aligned in one row and the simple pattern the members aligned in two rows.

From the data we got, splitting is the main strategy for variation when groups passed by the obstacle. $94.1 \%$ of free pattern groups and $83.2 \%$ of simple pattern groups split into two clusters to go through the sidewalks no matter how wide the obstacles are. But a significant difference occurs with the splitting strategy between the two patterns. When free pattern groups pass by the obstacle, the main strategy of splitting is to keep the configuration of the subgroups. It is observed that $84.3 \%$ of the subgroups keep their configuration while splitting, $9.2 \%$ of them vary their configuration and only $7.1 \%$ split and regroup into new subgroups. When simple pattern groups pass by the obstacle, the main strategy of splitting is to split the subgroups. $73.2 \%$ of the complex groups split their subgroups when passing a $0.6 \mathrm{~m}$ wide obstacle and $67.3 \%$ split their subgroups when passing a $1.2 \mathrm{~m}$ wide obstacle.

Although the splitting strategies are different, almost all subgroups return to their original configuration after passing the obstacles and most of the complex groups reorganize to their original structure no matter which strategy they choose for passing by the obstacles. Fig. 8 shows the examples of splitting strategies of most of the free pattern and simple pattern complex groups.

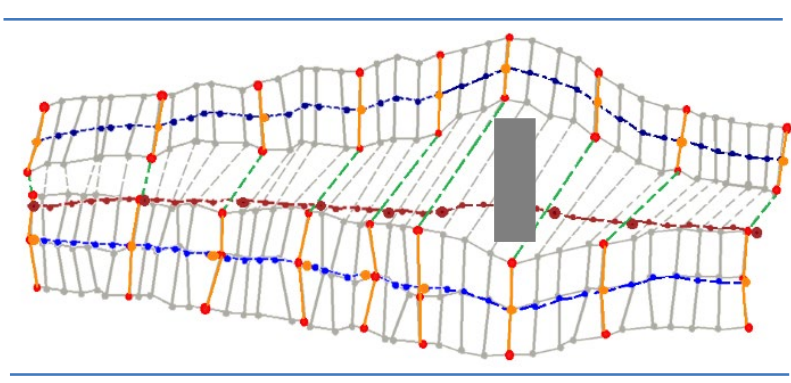

(a) splitting strategies of most of the free pattern

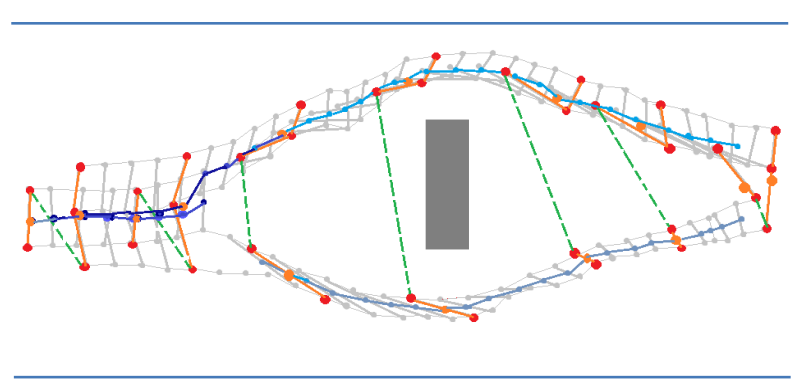

(b) splitting strategies of most of the simple pattern Fig.8: Variation Strategies in obstacle avoidance

\subsection{Variation Strategies When Facing a Counter Group}

In scene 3, two complex groups with 5-7 members walked oppositely from in the passage and passed by each other. 46 groups were tested in a $5 \mathrm{~m}$ wide passage and 44 tested in a $3 \mathrm{~m}$ wide passage. As shown in Table 1, most groups choose passing through or finger cross and break the group configuration while keeping less than half of the configuration of subgroups.

Table 1: Passing and Variation Strategies for counter groups

\begin{tabular}{|c|c|c|c|c|c|}
\hline \multirow{3}{*}{$\begin{array}{l}\text { Passage } \\
\text { width }\end{array}$} & \multicolumn{3}{|c|}{ Passing Strategies } & \multicolumn{2}{|c|}{ Variation Strategies } \\
\hline & Passing round & Passing through & Finger cross & Breaking group & Breaking subgroup \\
\hline & 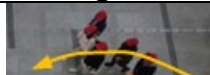 & & & & \\
\hline $3 \mathrm{~m}$ & $18.8 \%$ & $25.0 \%$ & $56.2 \%$ & $73.7 \%$ & $31.8 \%$ \\
\hline $5 \mathrm{~m}$ & $5.3 \%$ & $10.5 \%$ & $89.5 \%$ & $92.3 \%$ & $50.7 \%$ \\
\hline
\end{tabular}

\section{Mutual influence between the Groups on the Crowd}

In scene 1, crowds with complex groups of 5-7 members were asked to walk through the narrowing passage (Fig. 1(b)) for 5 times as well as the crowds with only individuals for contrast. In scene 2, crowds with complex groups of 5-7 members were asked to walk through the passage and pass by the obstacle (Fig. 2(b)) for 10 times as well as the crowds with only individuals. In scene 3, counter flows and vertical 
crossing flows with complex groups and with only individuals were studied. In this way, we wanted discover the mechanism of mutual influence between the Groups on the Crowd in conflict conditions.

\subsection{Influence of the Crowd on the Groups in Strategies Choosing}

Variation strategies of the groups are affected by the crowd. In scene 1, the proportion of keeping constant subgroup members is $78.9 \%$, which is quite similar to the single group experiments. But because of the disturbance and influence from other groups and individuals, it is much more difficult for the groups to keep configuration of the whole group and subgroup. $73.3 \%$ complex groups change their configuration and $78.1 \%$ of the subgroups change their configuration. In scene 2, the proportion of group splitting is $82.6 \%$, which is similar to the single group experiments. And $81.4 \%$ of the subgroups change their configuration because of the influence of the crowd. In both scenes, the variation of the complex and subgroups happen simultaneously and the group members arrange in the complicated pattern.

The results show that the groups do not have significant effect on crowd evacuation in narrow passage and bottle neck in respect of evacuation time. The evacuation time for crowds composed with groups and with only individuals are nearly the same, approximately 27-29s in scene 1 and 20 s in scene 2 .

Groups do not have obvious effect on crowd in sidewalk choosing when passing by the obstacle, but it seems that single groups make slightly better decision than the crowds do. When groups pass by the obstacle in sequence, the linear correlation between the pedestrian number and sidewalk width are stable and obvious, for instance, $33.5 \%$ of the members choose the narrower sidewalk which has $1 / 2$ width of the wider one. While the crowds pass by the obstacle, $40.7 \% \%$ of the pedestrians choose the narrower sidewalk in different experiment rounds, which shows that single groups could make better decision than the crowd to split into the sidewalks with a higher walking efficiency and lower density.

\subsection{Effect of Groups on the Self-organization}

Groups have significant effect on self-organization in counter flows by decrease the number of lanes and widen each lane. In a $6 \mathrm{~m}$ wide passage, 60 people were divided into two clusters to form counter flows. In counter flows with groups, 3-5 lanes were observed while 8-10 lanes were observed in counter flows with only individuals (See Fig. 9). The data in $3 \mathrm{~m}$ wide passage was 3-4 vs 4-5. Groups increase the average width of the lanes from $0.7 \mathrm{~m}$ to $1.5 \mathrm{~m}$. It is precisely the width of a 3 -member subgroup (see reference [5]). So the subgroups following in rear of a lane can keep its configuration better.

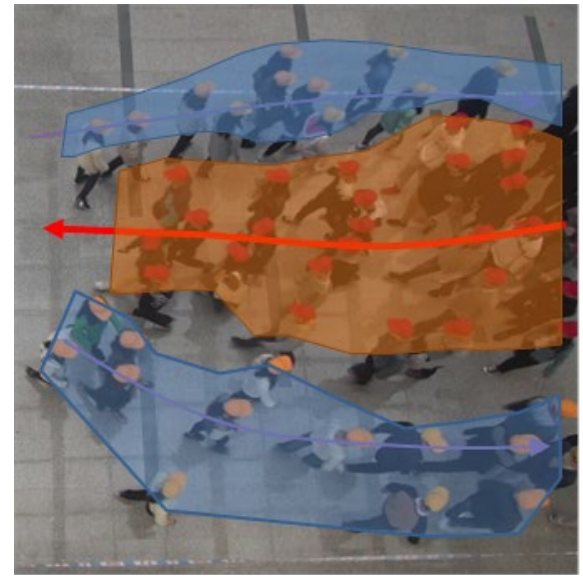

(a) Counter flows with groups

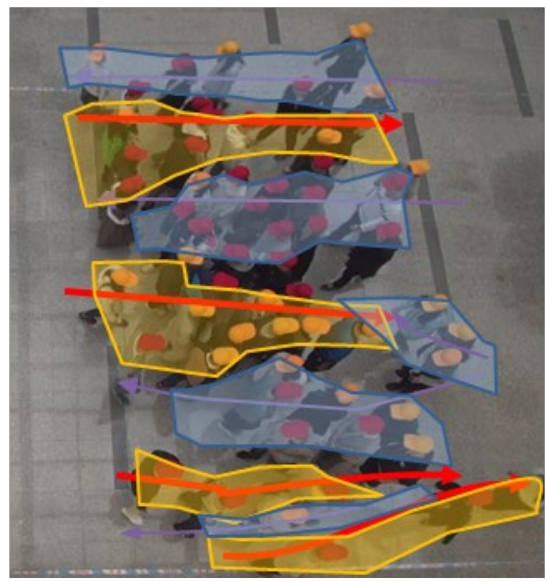

(b) Counter flows with individuals

Fig.9: Effect of groups on counter flows

Groups also have significant effect on the self-organization in vertical cross flows by decrease the number of lanes and widen each lane at the cross. Groups increase the average width of the lanes from $0.6 \mathrm{~m}$ to $1.3 \mathrm{~m}$. It is approximately the width of a subgroup with 2-3 members. 


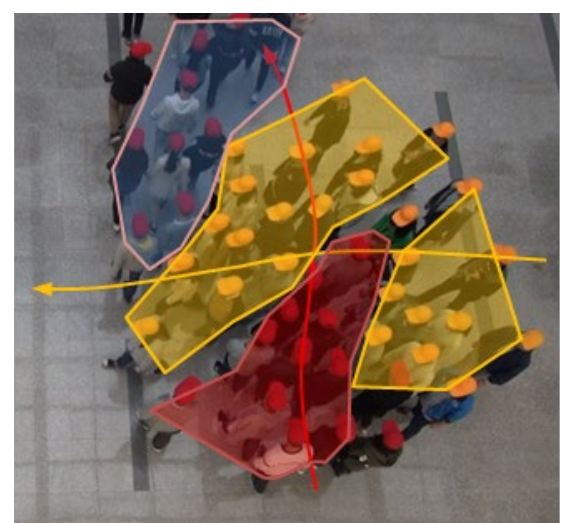

(a) Vertical cross flows with groups

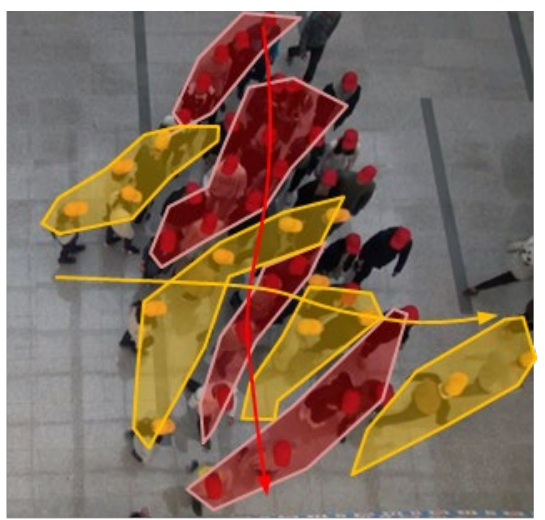

(b) Vertical cross flows with individuals

Fig.10: Effect of groups on vertical cross flows

\section{Conclusion}

In this paper, we studied the variation strategies of complex groups as well as the mutual influence between the groups and the crowd when they walked through a narrowing passage. The strategies of keeping configuration of complex groups and subgroups in narrowing passage, the strategies of splitting the groups when passing by the obstacle, and the strategies of breaking the groups and reorganizing could be applied in modelling pedestrian group decision and behavior. The study of the effect of groups on self-organization of the crowd is new in this field.

More work should be done in comparison of the walking velocity and evacuation efficiency under different strategies. Whether the effect of groups on self-organization is positive for crowd evacuation should be tested with detailed dynamic analysis.

\section{Acknowledgements}

The work was supported by Natural Science Foundation of China, Grant No. 51208381, and Scientific Research Funding for Youth Science Fund Project. The work was also supported in part by Natural Science Foundation of China, Grant No. 71271153. The key laboratory of road and traffic engineering of the Ministry of Education provided the support for the experiments. We are grateful to Jiajun Huang, Mengru $\mathrm{Xi}$ and the students from Tongji University for conducting and participating in the experiments.

\section{References}

[1] Jia'an Xi, Xiaolei Zou, Zhuo Chen, Jiajun Huang, Multi-pattern of Complex Social Pedestrian Groups, Transportation Research Procedia 2, 2014: pp. 60-68.

[2] Jiajun Huang, Xiao-lei Zou, Xiaoyi Qu, Jie Ma, Ruihua Xu. A Structure Analysis Method for Complex Social Pedestrian Groups with Symbol Expression and Relationship Matrix, Conference of Pedestrian Evacuation Dynamics 2016: pp. 283-289.

[3] Francesco Zanlungo, Takayuki Kanda. Do walking pedestrians stabily interact inside a large group? Analysis of group and sub-group spatial structure. Proceedings of the Annual Meeting of the Cognitive Science Society 2013: pp. 3847-3852.

[4] M. Moussaïd, N.Perozo, S. Garnier, D. Helbing, and G. Theraulaz The walking behaviour of pedestrian social groups and its impact on crowd dynamics. PLoS ONE, 2010, 5(4): e10047.

[5] Xiaolei Zou, Xiaoyi Qu, Ruihua Xu. Data-driven modeling on spatial pattern and private space of pedestrian groups. Proceedings of The 11th International Conference of Chinese Transportation Professionals. 2011: pp. 2102-2108. 